\title{
Elster-Löß und Holstein-Ton von Northeim (Hann.)
}

\author{
Von Gerd Lüttig, Hannover
}

Mit 1 Abbildung im Text

I n ha l t. Unter dem Holstein-Interglazialton der Ziegelei Kerrl in Northeim, das zur Zeit relativ gut aufgeschlossen ist, wurde elstereiszeitlicher Löß aufgefunden. Da ein sicherer Nachweis eines Lösses solch hohen Alters noch nicht erbracht worden ist, wird der neue, auch für die Beurteilung des Interglazials wichtige Aufschluß kurz beschrieben.

$\mathrm{S} u \mathrm{~m} m$ a r y. Elster-glacial loess has been found below the Holstein Interglacial clay of the brick-works Kerrl at Northeim. This formation is now rather well exposed. Up to the present, there has not been safe evidence of loess of such a high age. A brief description of this new outcrop which is of significance for the Interglacial as well, is therefore presented.

\section{1.}

Im Jahre 1928 (a, b) beschrieb H.-L. Heck den Aufschluß der Kerrl'schen Ziegelei in Northeim (Blatt Nörten, 4325, der topographischen Karte 1:25000) wie folgt:

$0,3 \mathrm{~m}$ verlehmte Schuttzone (teils Ackerboden)

$1,5-2,0 \mathrm{~m}$ gelb-braune tonige Sande mit vie'en und verschiedensten Geröllen

$1,5 \mathrm{~m}$ grauer-gelber sandiger Ton mit kleinen Geröllen

über $2,0 \mathrm{~m}$ dunkler zäher Ton mit Vivianit und Holzresten.

Der damalige Aufschluß lag in der Nähe von Punkt re 3568805 h 5729820 . Nach HECKs pollenanalytischer Untersuchung sind die drei liegenden Horizonte während einer Birken-Fichtenzeit und einer Eichenmischwaldzeit (von unten nach oben) abgelagert worden. In den tonigen Sanden, die nach den neuen Beobachtungen in einen wesentlich jüngeren geologischen Zeitraum gehören, fanden sich nur Pollen von Betula.

Wegen des großen Probenabstandes und der Fortschritte, die die Palynologie inzwischen gemacht hat, wäre eine neue pollenanalytische Untersuchung des Profils notwendig. Eine bei Prof. Firbas, Göttingen, angeregte Untersuchung durch S. Chanda (1960) in einem nahe Punkt re 68790 h 29790 gelegenen Profil erbrachte einen Fund von Azolla filiculoides Lam., durch den, wie durch die pollenanalytische Untersuchung, die Einstufung des Tones in das Holstein-Interglazial, wie sie durch $\mathrm{HECK}_{\mathrm{E}}$ und den Verfasser (LüтTIG 1954 b) vorgenommen wurde, ihre Bestätigung findet.

\section{2.}

Das Interglazial von Northeim wurde im Jahre 1954 südöstlich Bahnhof Northeim erbohrt (Bohransatzpunkt I re 68190 h 29560). Das Profil dieser Bohrung ist in Band 4/5 dieser Zeitschrift auf Seite 24 wiedergegeben, so daß sich eine genaue Wiedergabe erübrigt. Wichtig sind folgende Schlußfolgerungen aus diesem Befund:

a) Der interglaziale Ton liegt hier in einem wesentlich tieferen Niveau als in der K.ERRL'schen Ziegelei und ist in bezug auf den gleichen Horizont in der Tongrube tektonisch versenkt. Diese Einsenkung (oder Hebung des Tones in der Ziegelei) folgt ausweislich von Kartierergebnissen auf Blatt Northeim den alten, meist rheinisch streichenden Störungen in der Tiefscholle des Leinetalgrabens.

b) Ư b e r dem interglazialen Ton folgt nach einer Sandlage zunächst eine Fließerde mit viel Keupermaterial, die nach oben zunächst toniger, dann wieder kiesiger wird, wobei im hangenden Teil Muschelkalkmaterial zunimmt. Diese Fließerde leitet die Sedimentation der Mittelterrasse ein und ist einer Kaltzeit zu Beginn der Saaleeiszeit zuzuordnen, die Northeimer Phase genannt worden ist (LütTIG 1958).

Darüber folgt der Mittelterrassenkörper, über den sich eine zweite Fließerde, im liegenden Teil mit viel ortsnahen (Lias), im hangenden Teil von weiter her antransportierten Geröllen (Muschelkalk), legt. Darüber folgt Flugsand und Löß der Weichseleiszeit. 
U n t e r dem Interglazialton sind grobklastische Sedimente bemerkenswert, die früher auch in der Tongrube aufgeschlossen gewesen sein sollen und von HECK (1928) der Oberterrasse gleich gesetzt worden sind, was aber sicher nicht richtig ist (LÜTTIG 1954 a, b). Darunter kommen hellblaugraue glimmerige und dunkelviolettbraune Tone vor, deren Ahnlichkeit mit dem elstereiszeitlichen Ton von Bilshausen (LüTTIG \& REIN 1954) bereits betont worden ist. Im Liegenden dieser Tone traf die Bohrung fluviatil transportierte Staubsande, darunter Mittleren Keuper an.



90

80

70
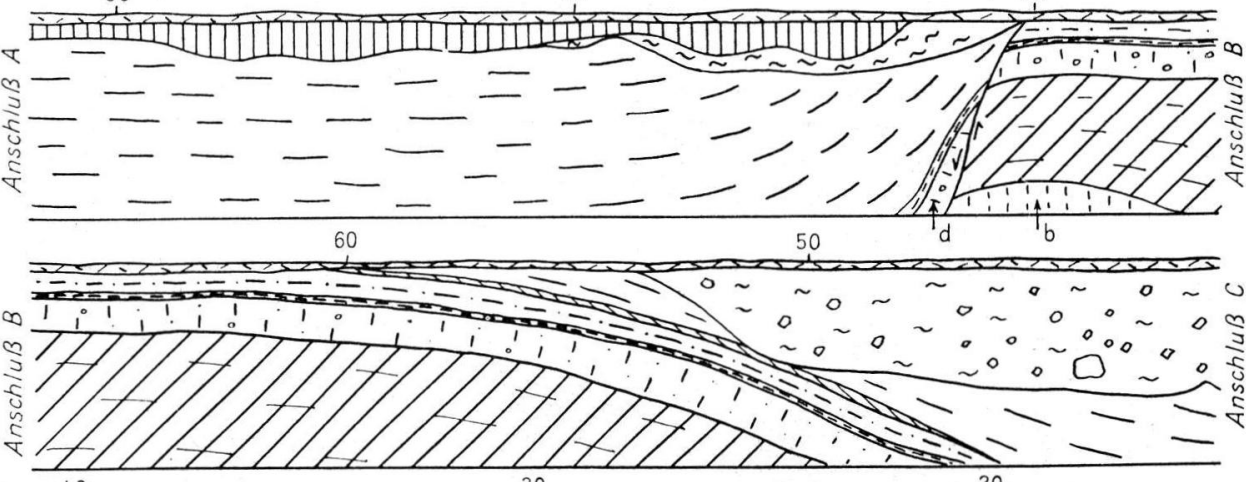

40

30

20

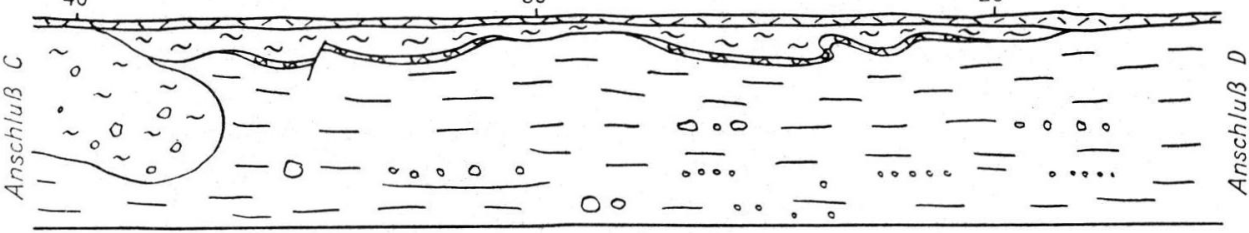

10

$\mathrm{Om}$

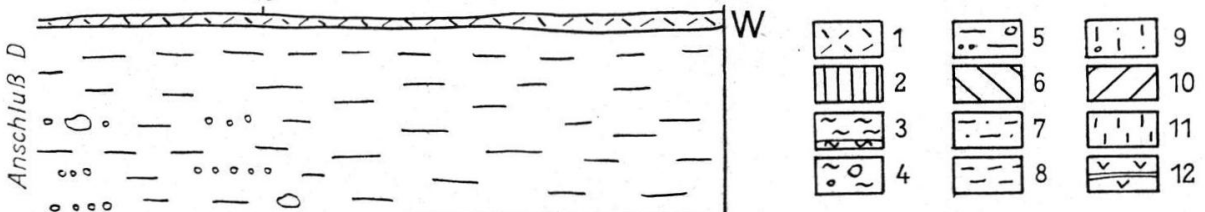

Abb. 1. Profil der Kerrlschen Ziegeleigrube Northeim.

$1=$ anthropogen gestörter Zersatz; $2=$ Lößlehm; $3=$ zweite lehmige Fließerde, blaugraubraun, an der Basis violettbraune Lage aus Jura-Schiefertonbrocken; 4 = erste kiesige Fließerde, violettbraun, Gerölle aus Rhätsandstein und Brauneisennester; $6=$ dunkelblaugrauer Ton; $7=$ mittelhellbrauner Schluff; 8 = hellblauer Ton; $9=$ rötl.-gelber, lößfarbener Lehm mit kleinen Geröllen und vereinzelten Holzkohlenresten, stark verfestigt; $10=$ rotbrauner sandig-schluffiger Ton; $11=$ hellbrauner -gelber sandiger Löß; $12=$ Mittlerer Keuper $=$ weinroter sandiger Schieferton mit einzelnen graugrünen Lagen, oben verwürgt. 
3.

Interessant ist nun in vielerlei Hinsicht ein neues Profil, das im Südstoß der Tongrube 1954 vom Verfasser aufgenommen worden und in Abb. 1 wiedergegeben worden ist. Es verläuft parallel dem südlich der KerRL'schen Tongrube vorüberführenden Wege. Der östliche Anfangspunkt des Profiles liegt beim Punkt re 68900 h 29770, seine Höhe (höchster Punkt des Profiles) dürfte etwa $+141 \mathrm{~m}$ NN entsprechen.

Im Ostteil der Aufschlußwand stehen unter 0,8 m Mutterboden und Lößlehm (höchstwahrscheinlich Weichsel-Löß) ebenliegende weinrote sandige Schiefertone des Mittleren Keupers mit einzelnen graugrünen Lagen (Rote Wand) an. In ihrem hangenden Teil sind diese Schiefertone deutlich verwürgt. Gegen den Mittleren Keuper sind an einer rheinisch streichenden, ca. $70^{\circ}$ nach W einfallenden Störung hellblaugraue schluffige Tone abgeschoben. Diese Tone repräsentieren das Interglazial von Northeim. In der Nähe der Störung, die mit der heutigen östlichen Verbreitungsgrenze des Tones ident ist, fällt der Ton zunächst relativ steil nach W (infolge der Abschiebung), das Einfallen verflacht sich aber dann recht bald. Zwischen Meter 110 und 70 des in Abb. 1 dargestellten Profiles ist der Ton relativ gleichkörnig, wird aber in westlicher Richung gröber, und es schalten sich einzelne Kieslagen ein, die in Schnüre aufgelöst erscheinen und z. T. recht grobe Blöcke enthalten.

In der Nähe von Meter 70 versteilt sich das Einfallen wegen einer steil nach E einfallenden Abschiebung auf kurzen Raum recht schnell. Abgeschoben ist die östlich der Störung gelegene Scholle. In der Nähe, und zwar östlich der Störung, kommt als Liegendschicht des Interglazials eine markante hellblaue Tonlage heraus. Unter dieser Tonlage steht Schicht d an, ein rötlich-gelber, lößfarbener Lehm mit einzelnen kleinen Geröllen und Holzkohlenresten. Diese Lage ist stark verfestigt. Eine von Herrn Dr. K. H. Sindowskr, Hannover, angefertigte Korngrößenanalyse ergab folgende Kornzusammensetzung:

\begin{tabular}{|c|c|c|c|c|c|}
\hline \multicolumn{3}{|c|}{ Siebanalyse: } & \multicolumn{3}{|c|}{ Pipettanalyse: } \\
\hline 15 & -5 & $1,6 \%$ & $200-100$ & $\mu$ & $11,3 \%$ \\
\hline 5 & -2 & $0,5 \%$ & $100-60$ & $\mu$ & $10,9 \%$ \\
\hline 2 & -1 & $0,3 \%$ & $60-30$ & $\mu$ & $12,8 \%$ \\
\hline 1 & $-0,6$ & $0,3 \%$ & $30-20$ & $\mu$ & $17,5 \%$ \\
\hline 0,6 & $-0,3$ & $0,7 \%$ & $20-10$ & $\mu$ & $7,2 \%$ \\
\hline $0, j$ & $-0,2$ & $1,2 \%$ & $10-6$ & $\mu$ & $3,0 \%$ \\
\hline 0,2 & $-0,12$ & $2,6 \%$ & $6-2$ & $\mu$ & $9,4 \%$ \\
\hline 0,12 & $-0,06$ & $1,4 \%$ & $<2$ & $\mu$ & $27,9 \%$ \\
\hline & $<0,06$ & $91,4 \%$ & & & \\
\hline
\end{tabular}

Nach der Korngrößenverteilung wie nach dem makroskonisch erkennbaren Sediment-

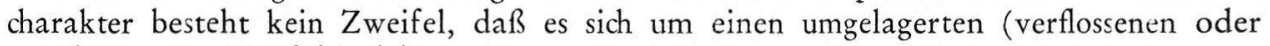
verschwemmten) Löß handelt.

Westlich der Störung bei Meter 70 sind zwischen diesem Schwemmlöß und dem hellblauen Ton einerseits und dem Interglazialton noch zwei Schichten entwickelt, die östlich der Störung, wohl weil diese syngenetisch zum Interglazialton ist, abgetragen sind. Es handelt sich hierbei um eine Lage dunkelblaugrauen Tons (unmittelbares Liegendes des Interglazials) und einen mittel- bis hellbraunen Schluff. Letzter bildet das Hangende des hellblaugrauen Tones über Schicht $d$.

Unter Schicht d kommt zwischen Meter 70 und 50 ein rotbrauner bis schokoladenbrauner sandig-schluffiger Ton heraus. Dieser Ton ähnelt petrographisch dem Elster-Ton von Bilshausen, der dort ebenfalls unter blaugrauem Schluff-Ton (von LütTig \& REIN 1954 gleichermaßen als Holstein-Interglazial aufgefaßt) liegt. Unter diesem rotbraunen Ton steht nun eine Schicht hellbraunen-gelben sandigen L ös se s an (Schicht b). Daß es sich um einen Fluglöß handelt, wird aus der folgenden Korngrößenverteilung sehr wahrscheinlich. 


\begin{tabular}{lrr}
\multicolumn{3}{c}{ S i e b a n a lyse: } \\
$1-0,6 \mathrm{~mm}$ & $0,2 \%$ \\
$0,6-0,3 \mathrm{~mm}$ & $0,9 \%$ \\
$0,3-0,2 \mathrm{~mm}$ & $1,7 \%$ \\
$0,2-0,12 \mathrm{~mm}$ & $2,4 \%$ \\
$0,12-0,06 \mathrm{~mm}$ & $1,2 \%$ \\
& $\langle 0,06 \mathrm{~mm}$ & $93,6 \%$
\end{tabular}

\begin{tabular}{rr}
\multicolumn{2}{c}{ P i pet t a n a ly s : } \\
$200-100 \mu$ & $13,7 \%$ \\
$100-60 \mu$ & $1,1 \%$ \\
$60-30 \mu$ & $21,3 \%$ \\
$30-20 \mu$ & $17,8 \%$ \\
$20-10 \mu$ & $7,5 \%$ \\
$10-6 \mu$ & $7,4 \%$ \\
$6-2 \mu$ & $8,9 \%$ \\
$<2 \mu$ & $22,3 \%$
\end{tabular}

Bei und westlich Meter 50 sind im Hangenden des Interglazialtones noch zwei verschiedene Fließerden (Saale- bis Weichselglazial) aufgeschlossen, die aber in diesem $\mathrm{Zu}$ sammenhang nicht näher zur Erörterung stehen sollen. Die ältere dieser Fließerden ist grobkörnig und enthält viel Rhätsandstein- und Lias-Gerölle, wohl von der Liasscholle im Verband der Leinetalgraben-Füllung oberhalb des Interglazialvorkommens. Die obere Fließerde ist feinerkörnig und enthält fast nur Gerölle aus jurassischem Tonschiefer. Ein Vergleich mit den in der Bohrung I (LüTTIG 1954 b) erbohrten Fließerden unter und über dem Mittelterrassenkies ist vorerst nicht möglich. Festgehalten werden muß, daß eine Bedeckung des Interglazialtones durch Mittelterrassenkies hier nicht beobachtet wurde. Auch die von HEcK (1928a) beschriebenen Kieslagen ließen sich nicht wiederfinden, waren aber vor einigen Jahren zu sehen. Es handelt sich dabei um sehr kleine linsenartige Vorkommen, die vermutlich in das Hangende von Schicht $d$ gehören.

4.

Die im Liegenden des Interglazialtones von Northeim aufgeschlossenen Schichten enthalten keine Fossilien. $\mathrm{Da}$ die Flora im Interglazialton vom liegenden zum hangenden Teil nach HECK (1928b) eine Klimaverbesserung kundzugeben scheint, können die unter dem Ton liegenden Schichten, falls die Zeit ihrer Ablagerung nicht durch eine längere Emersionsphase vom Interglazial getrennt ist, in dic voraufgehende Kaltzeit, die ElsterEiszeit gestellt werden. Es kann sicherlich mit kleineren Emersionszeiträumen zwischen der Ablagerung der einzelnen Horizonte gerechnet werden. Nach dem Bild, das man von der Sedimentation der im Profil aufgeschlossenen Schichten gewinnt, kann man aber größere Schichtlücken ausschließen. Außerdem kommen im Liegenden des Interglazialtones verschwemmter Löß (Schicht d) und Fluglöß (b) vor, die auf eine Kaltzeit hindeuten. Der rote Ton zeigt an, daß organische Substanz im Gewässer, in dem er zur Ablagerung kam, nicht in dem Maße vorhanden war, daß der Sauerstoff im Wasser aufgezehrt werden konnte. Es muß deshalb im Gewässer gute Belüftung geherrscht haben, die zur Oxydation des Eisens führte bzw. eine Reduktion zum zweiwertigen Eisen, welches wie im Interglazialton blaugraue Färbung des Sedimentes bewirkt hätte, verhinderte.

Alle diese Eigentümlichkeiten der Schichten unter dem Interglazialton deuten darauf hin, daß sie während einer $\mathrm{K}$ altze it abgelagert worden sind. Nach Lage der Dinge kann aber diese Kaltzeit nur der Els te re is z e it entsprechen.

Wenn dieser Schluß richtig ist - nach Auffassung des Autors kann daran kein Zweifel bestehen -, dann haben wir es bei Schichten b und d mit elstereiszeit lichem $\mathrm{L}$ öß zu tun. Das ist ein immerhin seltener Fall, der m. E. Erwähnung verdient.

In unserem Gebiet ist bisher nur einmal (Lürtig 1954c) aus dem Raum von Lamspringe umgelagerter Elster-Löß erwähnt worden. Elster-Löß unter Saale-Grundmoräne beschrieb von Crinitz in der Niederlausitz auch ZöLLER (1934). Er nannte ihn „älteren Löß“, womit allgemein saaleglazialer Löß gemeint wird. Falls der Löß nicht, wie Woldstedt (1950) vermutete, doch in die frühe Saaleeiszeit gehört, müßte man freilich die Bezeichnung „ältester Löß“ verwenden (OLBRicht 1922). M. E. sollte man aber die Ausdrücke „jüngster“, „jüngerer“, „älterer“ und ,ältester“ Löß überhaupt vermeiden. 
Für von Picard (1950) aus dem Stadtgebiet von Essen unter Saale-Grundmoräne beschriebenen „älteren Löß“ gilt das gleiche wie für den ZöLLER'schen Löß. Alle diese Lösse lassen die Möglichkeit offen, daß sie noch ins Saaleglazial gehören könnten. Nicht so die Lösse von Northeim, die zweifelsfrei älter sind.

6.

Auch bezüglich der jungen Tektonik des Leinetalgrabengebietes besagt der Aufschluß einiges Neue. Zum ersten ist die Ostrandstörung des Interglazialvorkommens (LüTrIG 1954a) nunmehr aufgeschlossen. Zum zweiten ist erwiesen, daß auch in der Tongrube Störungen das Interglazial in sich selbst betreffen. Für die ehemalige Lage der Interglazialbasis bedeutet dies aber, daß sie höher gewesen sein muß als $+125 \mathrm{~m} \mathrm{NN}$. In Bohrung Northeim I wurde sie bei ca. $+113 \mathrm{~m}$ angetroffen, liegt aber dort in einer Scholle, die stark eingesenkt liegt.

Die ursprüngliche Lage der Basis und damit auch der Untergrenze der alten Taleintiefung ist damit schwer zu ergründen; denn weder das Gebiet der Ziegelei noch um den Bahnhof Northeim lag tektonisch ruhig. So kommt es auch, daß in diesem Raume FlsterSedimente, die normalerweise höher am Hang liegen als die Saaleablagerungen, hier das Liegende der Holstein-Tone bilden, daß aber andererseits Holsteintone höheren Talauen-Abstand besitzen als der Mittelterrassenkörper, in dessen Liegendes sie normalerweise gehören.

Nur in tektonisch labilen Gebieten kann sich, wie hier, der Flachlands-Lagerungstyp des Quartärs (ältestes zu unterst) neben dem Berglandtyp (jüngste Ablagerung mit dem geringsten, älteste mit dem höchsten Talauen-Abstand) ausnahmsweise einstellen. Da aber die Lagerung der Quartärablagerungen im Gebiet von Northeim stellenweise abnorm ist, scheidet das Gebiet für die Konstruktion eines Erosions- und Akkumulations-Diagrammes (Heck 1928 a, Abb. 5, Seite 450) als ungeeignet aus.

\section{Schrift tum :}

CHANDA, S.: Über spättertiäre und interglaziale, pollenführende Ablagerungen im Leinetal nahe Göttingen und im südwestlichen Harzvorland (Unter-Eichsfeld). - Die Naturwiss. 47, 19-20, Berlin - Göttingen - Heidelberg, 1960.

HECK, H.-L.: Beiträge zur Talgeschichte der oberen Leine. - Jb. pr. geol. L.A. f. 1928, 49, 427 bis 453, Berlin 1928. - - Pollenanalytische Untersuchungen altdiluvialer Tone und Torfe von Northeim und Eichenberg im Flußgebiet der Leine. - Jb. pr. geol. L. A. 49, II, 1255-1264, Berlin 1928 (1928b).

Lürtig, G.: Pleistozän-Tektonik nördlich Northeim. - Geol. Jb. 68, 587-614, Hannover 1954(a). - - Klima und Tektonik des Pleistozäns von Northeim. - Eiszeitalter u. Gegenwart 4/5, 22-33, O'hringen/Württ. 1954(b). - - Alt- und mittelp!eistozäne Eisrandlagen zwischen Harz und Weser. - Ungedr. Dissertation, Göttingen 1952. Geol. Jb. 70, 43-125, Hannover 1954(c). - - Eiszeit-Stadium-Phase-Staffel. Eine nomenklatorische Betrachtung. Geol. Jb. 76, 235-260, Hannover 1958.

OlbRicht, K.: Die Eiszeit in Deutschland und der vorgeschichtliche Mensch. - Naturw. Wochenschr. 21, 369-381, Jena 1922.

Picard, K.: Beobachtungen im Diluvium des Stadtgebietes Essen. - Jb. geol. L. A. 65, 573-587, Hannover 1950.

Woldstedt, P.: Norddeutschland und angrenzende Gebiete im Eiszeitalter. - 1. Aufl. Stuttg. 1950.

Zöller, A.: „Alterer“ Löß bei Crinitz i. d. Niederlausitz (Meßtischbl. Fürstlich-Drehna). - Z. deutsch. geol. Ges. 86, 235-238, Berlin 1934.

Manuskr. eingeg. 18. 11. 1960.

Anschrift des Verf.: Dr. Gerd Lüttig, Niedersächsisches Landesamt für Bodenforschung, Hannover, Wiesenstraße 1. 